\title{
Amoebic Colonic Perforation Presenting as Peritonitis in Emergency, Incidence and Outcome: Our Experience
}

\author{
Muzzafar ZAMANª , Kunal CHOWDHARYa, Gurinder KAUR ${ }^{b}$, Aliya SHAHc
}

a Department of Surgery, MMIMSR Mullana, Ambala Haryana 133207, India

bDepartment of Anaesthesia, MMIMSR Mullana, Ambala Haryana 133207, India

'Department of Microbiology, MMIMSR Mullana, Ambala Haryana 133207, India

\begin{abstract}
Objectives: Amoebiasisis a common parasitic infection and fulminant amoebic colitis remains a very rare complication of this disease. The objective of the present study was to find the incidence and outcome of perforation peritonitis due to amoebic colitis in MMIMSR, Mullana, Ambala, India.

Material and methods: 200 patients with perforation peritonitis were studied retrospectively, out of which 22 cases had colonic perforation, were selected over a period of five years, between 2012 to 2017, at MMIMSR Mullana. All patients had undergone emergency laparotomy and cases with amoebic colonic perforation established on histopathology were studied in terms of incidence, clinical presentation and outcomes.

Results: Out of 200 emergency exploratory laparotomies carried out during 2012-2017 at MMIMSR, Mullana, $22(11 \%)$ patients had amoebic colonic perforation. The disease was more common among men as compared to women (21:1). The mean age of patients was $60 \pm 10$ years. Symptom duration ranged from 1 to 8 days. $15(68 \%)$ patients had historyof chronic alcohol intake and $2(9 \%)$ had a recent episode of loose stools. Chest X-ray showed free gas under the diaphragm in $45 \%(10 / 22)$ of patients. Ultrasonography whole abdomen showing associated liver abscess was seen in 8/22 (36\%) patients. Intraoperatively caecal perforation was seen in 20 cases, out of which 16 had concomitant ascending colon perforation and 2 sigmoid colon perforation. Bowel resection was performed depending upon the site and extent of the colon involvedright hemicolectomy (16), limited ileocolic resection (4) and sigmoidectomy (2). Proximal diversion stoma was made in all cases. Postoperative complications encountered were wound infection in 20 (91\%) cases, followed by burst abdomen in $8(36 \%)$ cases, stoma related complication in $6(27 \%)$ cases. The overall mortality rate due to sepsis was $54 \%$ (12/22).

Conclusion: Although amoebiasis is a common parasitic infection, fulminant amoebic colitis remains a very rare complication with a reported incidence of $1 \%$. Even with aggressive management of this entity, patients have got a poor prognosis. Resection with exteriorization of bowel is the current gold standard treatment in such cases.
\end{abstract}

Keywords: colonic perforation, amoebic colitis, faecal peritonitis.

Address for correspondence:

Kunal Chowdhary, Assistant Professor Department of General Surgery, Maharishi Markandeshwar Institute of Medical Sciences and Research (MMIMSR), Mullana, Ambala Haryana 133207, India

Tel.: 8505993535

Email:kunal_k04@rediff.com

Article received on the $21^{\text {st }}$ of February 2018 and accepted for publication on the $12^{\text {th }}$ of March 2018. 


\section{INTRODUCTION}

Amoebiasis is a protozoal disease caused by Entamoeba histolytica. It is endemic in tropical countries and has become a health problem worldwide. It presents as a wide spectrum depicting pathology as benign as the asymptomatic carrier state to fulminant colitis and colonic perforation. With the widespread use of anti-amoebic drugs, very few cases of amoebic colonic perforation were reported in the literature especially over the past 20 years. This study was done to highlight the incidence and outcomes of amoebic colonic perforation as encountered by us at Maharishi Markendeshwar Institute of Medical Sciences \& Research (MMIMSR), MullanaAmbala India.

\section{MATERIALS AND METHODS}

Two hundred patients with perforation peritonitis were studied retrospectively, among which 22 cases with colonic perforation, were selected over a period of five years between 2012 and 2017, at MMIMSR, Mullana. All patients had undergone emergency laparotomy, and the cases with amoebic colonic perforation established on histopathology were studied in terms of incidence, clinical presentation and outcomes.

Inclusion criteria: All patients with amoebic colonic perforation confirmed on histopathology report.

Exclusion criteria: Patients with other causes of gut perforation causing peritonitis.

Case records of all patients who met the inclusion criteria were studied in terms of clinical presentation, radiological investigations, operative findings and postoperative course. Data was collected from indoor patient records, operation theatre records and outpatient department follow up of cases.

All cases diagnosed with perforation were aggressively resuscitated and built up for surgery. In all cases, exploratory laparotomy was done with vertical midline incision, site of perforation was seen and adequately managed, and peritoneal cavity was irrigated with warm normal saline till the returns were clear. The abdomen was closed with continuous number one non-absorbable suture material after inserting two drains (one subhepatic and the other one pelvic). Postoperatively, all patients received antibiotics, initially empirically and then according to the culture sensitivity reports of their peritoneal fluid.
No Human and Animal Rights have been violated in the present study.

Statistical analysis was not applicable in our study.

\section{RESULTS}

Out of 200 cases of emergency exploratory laparotomy carried out between 2012 and 2017, at MMIMSR, Mullana, 22 (11\%) patients had amoebic colonic perforation. The disease was more common among male subjects than among female subjects (21:1). The mean age of the patients was $60 \pm 10$ years. Symptom duration ranged from one to eight days. The number of patients with history of chronic alcohol intake was $15(68.18 \%)$, and two (9\%) had recent episode of loose stools. All patients presented with abdominal pain with obstipation.

Physical examination revealed abdominal distension with diffuse tenderness in the abdomen in all patients. Chest X-ray showed free gas under the diaphragm in $45.5 \%(10 / 22)$ of patients. UItrasound whole abdomen showing associated liver abscess was seen in 8/22 (36.6\%) patients. Intraoperatively caecal perforation was seen in 20 cases, out of which 16 had concomitant ascending colon perforation, and in two cases, sigmoid colon perforation was noticed. Bowel resection was performed depending upon the site and extent of the colon involved - right hemicolectomy (16), limited ileocolic resection (4) and sigmoidectomy (2), as shown in Figures 1, 2, 3. Proximal diversion stoma was made in all cases.

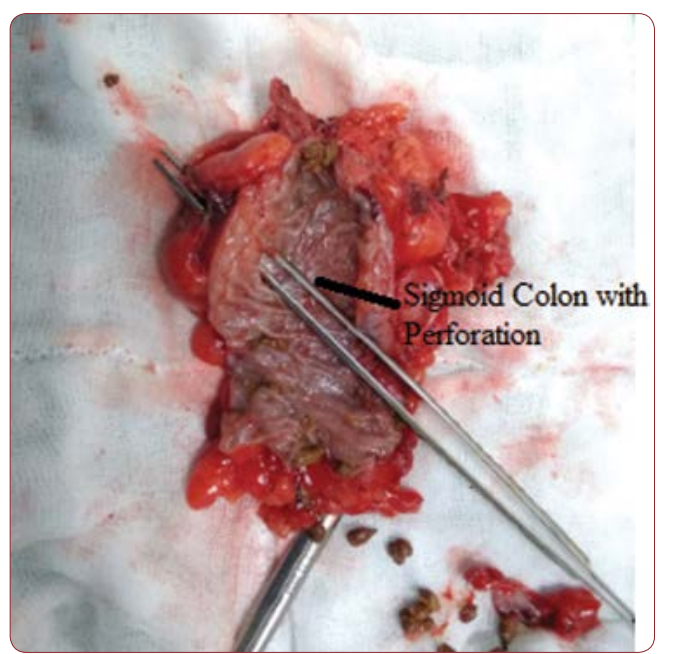

FIGURE 1. Resected specimen of sigmoid colon with perforation 


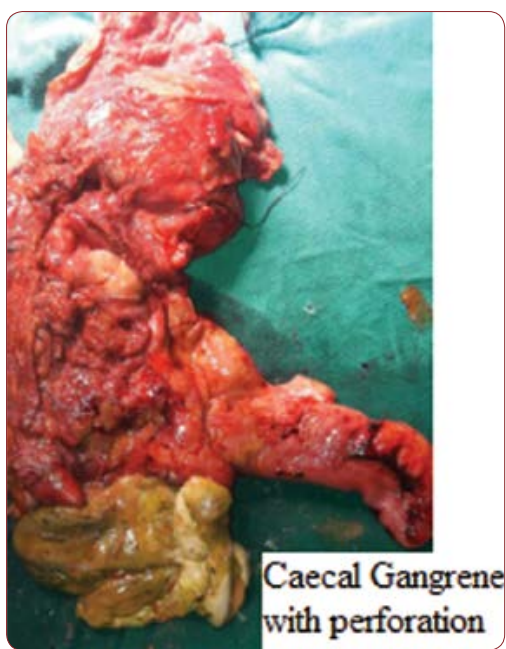

FIGURE 2. Resected specimen of right hemicolectomy with caecal and ascending colon perforation

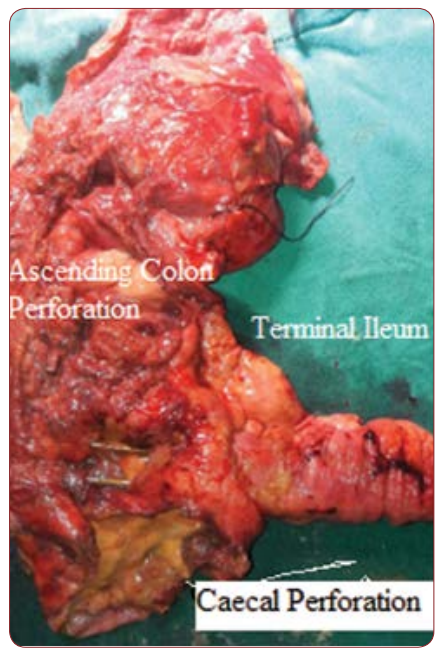

FIGURE 3. Resected specimen with caecal perforation

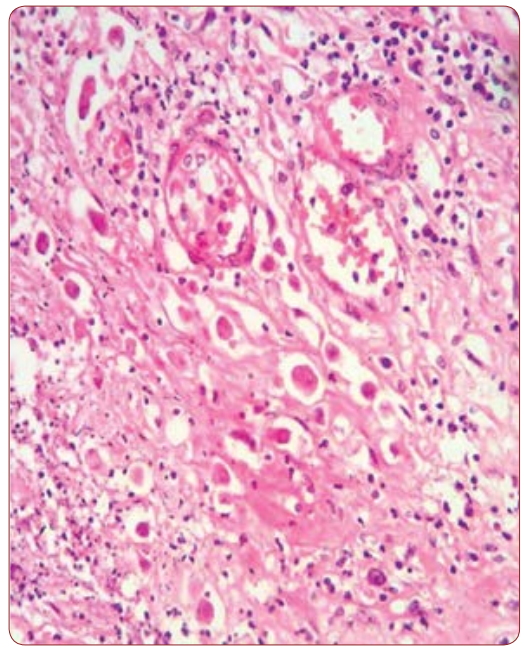

FIGURE 1. Resected specimen of sigmoid colon with perforation
Histopathology showed amoebic infection in all cases (Figure 4). Postoperative complications were wound infection in 20 cases $(90.9 \%)$, followed by burst abdomen in eight cases (36.36\%), and stoma related complication in six cases $(27.2 \%)$. Overall mortality rate due to sepsis was $54.5 \%(12 / 22)$.

\section{DISCUSSION}

Amoebiasis presents as a wide spectrum depicting pathologies as benign as the asymptomatic carrier state to fulminant colitis and colonic perforation. E. histolytica infections, which cause amoebiasis, begin with ingestion of water contaminated with fecal matter containing cysts of the amoeba (1). Trophozoites of the amoeba are released into the lumen of the gastrointestinal tract, where they rapidly clone themselves and further form new cysts, which are excreted in the faeces (2). The infection can be located in any part of the intestine, but is most likely to be found in the caecum and ascending colon, as seen in our case (3).

In our study, amoebic colonic perforation was seen in 22 patients, which is comparable to other studies with similar gender distribution. Patients with amoebic colonic perforation were $>40$ years old, most of them being in their fifth (40-49) to sixth (50-59) decade of life, which was in contrast to other worldwide published reports (3).

Preoperative clue to diagnosis by history of amoebic colitis was not a dependable parameter, as it was positive in only $9 \%$ of cases in our study, which agreed to findings reported by other authors (1).

Fulminant colitis, which is a known to be a variant of amoebic colitis, develops rapidly and presents with features of acute abdomen and loose stools. Various risk factors, including male gender, increased age, signs of peritonitis and abdominal pain, leukocytosis, electrolyte disturbances, and hypoalbuminemia, are associated with the development of fulminant amoebic colitis in patients with invasive intestinal amoebiasis $(4,5)$. In our study, associated liver abscess was seen in 36\% cases, emphasing its coexistence, and it was noticed that these patients were more sick and developed more postoperative complications compared to those who presented only amoebic colonic perforation. Most common perforation was seen in caecum, with a good num- 
ber of patients having multiple perforations involving caecum and ascending colon, which differed from the available studies, where ascending colon appeared to be the most common site $(1,2,4)$.

Our study highlighted the fact that the current available gold standard treatment was resection of the perforated bowel with exteriorizing the proximal bowel, as we did in all our patients, as the literature provided us adequate data showing that anastomosis of bowel had higher leak rates and elevated risk of suture breakdown in tissue containing amoebae $(1,6-9)$. Necrotizing amoebic colitis is associated with mortality ranging from $55 \%$ to $100 \%$ even with the best treatment, which is exactly what we found in our study $(8,9)$.

\section{CONCLUSIONS}

Fulminant amoebic colitis remains a very rare complication. Amoebic colitis causing colonic perforation incidence is higher in developing and third world countries, in which there is a poor overall health status and where portable water is not always available.

Even with aggressive management, the prognosis of such patients is poor. Resection with exteriorization of bowel is the current gold standard treatment in such cases.

Conflicts of interest: none declared.

Financial support: none declared.

\section{R}

1. Jain BK, Garg PK, Kumar A, Misra K, et al. Colonic perforation with peritonitis in amoebiasis:a tropical disease with high mortality. Trop Gastroenterol 2013;34:83-86.

2. Gupta SS, Singh O, Shukla S, Raj MK. Acute fulminant necrotizing amoebic colitis: A rare and fatal complication of amoebiasis: A case report. Cases J 2009;2:6557.

3. Takahashi T, Gamboa-Dominguez A, Gomez-Mendez TJ, Remes JM, Rembis V, Martinez-Gonzalez D, et al. Fulminant amebic colitis: Analysis of 55 cases. Dis Colon Rectum 1997;40:1362-1367.
4. Nisheena R, Ananthamurthy A, Inchara YK. Fulminant amebic colitis: A study of six cases. Indian J PatholMicrobiol 2009;52:370-373.

5. Athié-Gutiérrez $C$, Rodea-Rosas $H$, Guízar-Bermúdez C, Alcántara A, Montalvo-Javé EE. Evolution of surgical treatment of amoebiasis-associated colon perforation. J Gastrointest Surg 2010;14:82-87.

6. Ishida $\mathrm{H}$, Inokuma $S$, Murata $\mathrm{N}$, Hashimoto D, Satoh K, Ohta S. Fulminant amoebic colitis with perforation successfully treated by staged surgery: a case report. J Gastroenterol 2003;38:92-96.

7. Chen HT, Hsu YH, Chang YZ. Fulminant amoebic colitis: recommended treatment to improve survival. Tzu Chi Med J 2004;16:1-8.

8. Yadav D, Garg PK. Spectrum of Perforation Peritonitis in Delhi: 77 Cases Experience. Indian J Surgery 2013;2:133-137.

9. Haque MA, Maharjan SB, Choudhary J. Spectrum of perforation peritonitis 260 cases experience. Post-Graduate Medical Journal of NAMS 2010;2:135-137. 